\title{
Twisting of Tail End of the Reservoir Bag Malingering as Stuck Valve during Positive-pressure Ventilation
}

\author{
Poonam Kumari ${ }^{1}$, Amarjeet Kumar ${ }^{2}$, Chandni Sinha ${ }^{3}$, Ajeet Kumar ${ }^{4}$, Rajesh Kumar ${ }^{5}$
}

\begin{abstract}
The most commonly used open circuit is The Jackson Rees modification of the Ayre's T-piece (Mapleson-F system) in pediatric patients because it has low resistance and nominal dead space. Here, we report a case in which we used the Jackson Rees circuit with bag tail valve for ventilation in pediatric patient weight $12 \mathrm{~kg}$. During positive-pressure ventilation, we felt resistance and the patient was not ventilating. High pressure in the bag was being formed despite the valve was fully open. On inspection, we found out the cause was a twisted bag tail end as shown in Figure 1. We straighten the tail end of the bag, which aided in releasing air pressure and the patient started getting ventilation. The obstruction of the expiratory limb of the reservoir bag may be due to a stuck valve or due to twisting of its tail end. Due to high flexibility, twisting of the tail end of reservoir bag commonly found when it was held with tail end up position. Due to the twisting of the tail end of the reservoir bag, the expiratory limb becomes closed and high flow oxygen started going to the inspiratory limb. This may result in $\mathrm{CO}_{2}$ retention and barotraumas of the lungs if the obstruction was not released. We can prevent twisting of the tail end of the bag by keeping the Jackson Rees circuit in a vertical position with the tail end facing down.

Keywords: Airway obstruction, Pediatric, Reservoir bag.

The Journal of Medical Sciences (2020): 10.5005/jp-journals-10045-00155
\end{abstract}

Sir,

The most commonly used open circuit is The Jackson Rees modification of the Ayre's T-piece (Mapleson-F system) in pediatric patients because it has low resistance and nominal dead space. ${ }^{1}$

The Mapleson had derivative of five specific components of the breathing system, i.e., fresh gas flow, breathing tube, mask, reservoir bag, and expiratory valve. This system is named as Mapleson system and entitled A to E. ${ }^{2}$ Further, Wills et al. ${ }^{3}$ added the Mapleson $F$ system. This device connects a two-ended bag to the expiratory limb of the circuit; gas escapes via the "tail end" of the bag, which permits respiratory movements to be very easily seen and allows intermittent positive ventilation if required. The bag is, nevertheless, not necessary to the functioning of the circuit. Intermittent positivepressure ventilation (IPPV) can be given by closing the tail of the bag between a finger and thumb and pressing the bag or by using the bag-tail valve. ${ }^{4}$

Here, we report a case in which we used the Jackson Rees circuit with bag tail valve for ventilation in pediatric patient weight $12 \mathrm{~kg}$. During positive-pressure ventilation, we felt resistance and the patient was not ventilating. High pressure in the bag was being formed despite the valve was fully open. On inspection, we found out the cause was a twisted bag tail end as shown in Figure 1. We straighten the tail end of the bag, which aided in releasing air pressure and the patient started getting ventilation.

The E "bag-tail valve" employs an adjustable resistance and partial inflation of the bag that helps in the one-handed performance of IPPV. The volume of the reservoir tubing must approximate the patient's tidal volume. If the volume is much more then rebreathing may occur and if the volume is too less then ambient air may be entrained. To prevent rebreathing, the technique applied is that the minimum flow rate is $4 \mathrm{~L} /$ minute,
${ }^{1,3-5}$ Department of Anaesthesiology, All India Institute of Medical Sciences, Patna, Bihar, India

${ }^{2}$ Department of Trauma and Emergency, All India Institute of Medical Sciences, Patna, Bihar, India

Corresponding Author: Amarjeet Kumar, Department of Anaesthesiology, All India Institute of Medical Sciences, Patna, Bihar, India, Phone: +91 9570890646, e-mail: amarjeetdmch@gmail.com

How to cite this article: Kumari P, Kumar A, Sinha C, et al. Twisting of Tail End of the Reservoir Bag Malingering as Stuck Valve during Positive-pressure Ventilation. J Med Sci 2020;6(3):54-55.

Source of support: Nil

Conflict of interest: None

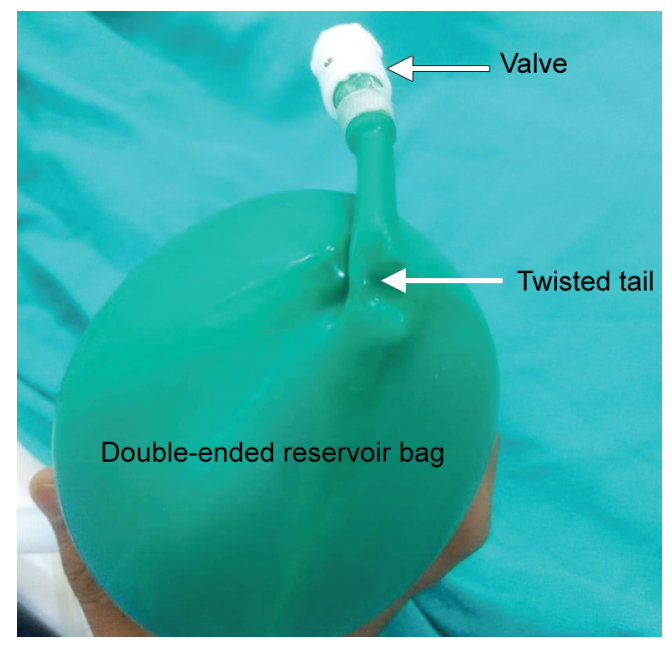

Fig. 1: Twisted tail end of the reservoir bag

(0) The Author(s). 2020 Open Access This article is distributed under the terms of the Creative Commons Attribution 4.0 International License (https:// creativecommons.org/licenses/by-nc/4.0/), which permits unrestricted use, distribution, and non-commercial reproduction in any medium, provided you give appropriate credit to the original author(s) and the source, provide a link to the Creative Commons license, and indicate if changes were made. The Creative Commons Public Domain Dedication waiver (http://creativecommons.org/publicdomain/zero/1.0/) applies to the data made available in this article, unless otherwise stated. 
with a fresh gas flow of 2.5-3 times the patient's minute volume. Advantages of the Jackson Rees circuit it is compact, inexpensive, marginal resistance to breathing, and cost-effective for controlled ventilation. The disadvantage of this circuit is the requirement of high gas flow. The bag can be distorted and inhibit breathing. The obstruction of the expiratory limb of the reservoir bag may be due to a stuck valve or due to twisting of its tail end. Due to high flexibility, twisting of the tail end of reservoir bag commonly found when it was held with tail end up position. Due to the twisting of the tail end of the reservoir bag, the expiratory limb becomes closed and high flow oxygen started going to the inspiratory limb. This may result in $\mathrm{CO}_{2}$ retention and barotraumas of the lungs if the obstruction was not released. We can prevent twisting of the tail end of the bag by keeping the Jackson Rees circuit in a vertical position with the tail end facing down.

\section{Consent}

Taken from the patient relative.

\section{References}

1. Jerwood DC, Jones SE. HME filter and Ayre's t piece. Anaesthesia 1995;50(10):915-916. DOI: 10.1111/j.1365-2044.1995.tb05873.x.

2. Mapleson WW. The elimination of rebreathing in various semiclosed anaesthetic systems. Br J Anaesth 1954;26(5):323-332. DOI: 10.1093/ bja/26.5.323.

3. Wills BA, Pender JW, Mapleson WW. Rebreathing in a T - piece: volunteer and theoretical studies of the Jackson - Rees modification of Ayres' T - Piece during spontaneous respiration. Br J Anaesth 1975;47(12):1239-1246. DOI: 10.1093/bja/47.12.1239.

4. Dorsch JA, Dorsch SE. Understanding anaesthesia equipment. Mapleson Breathing System. 5th ed., New Delhi: William and Wilkins; 2008. pp. 209-221. 